\title{
A rare magnetic resonance imaging pattern of leptomeningeal carcinomatosis: a case description
}

\author{
Chang Liu ${ }^{1,2,3 \#}$, Yu Zhang ${ }^{1,2,3 \#}$, Yi Li ${ }^{1,2,3}$, Hui Bu ${ }^{1,2,3}$, Zhaohua Mengi, ${ }^{1,2,3}$, Yingxiao Ji ${ }^{4} \mathrm{Jiang} \mathrm{Wu}^{5}$, \\ Yueli Zou ${ }^{1,2,3}$, Xinxin Chen ${ }^{1,2,3}$, Junying $\mathrm{He}^{1,2,3}$, Kun Hong ${ }^{1,2,3}$ \\ ${ }^{1}$ Department of Neurology, The Second Hospital of Hebei Medical University, Shijiazhuang, China; ${ }^{2}$ Institute of Cardiocerebrovascular Disease, \\ Shijiazhuang, China; ${ }^{3}$ Neurological Laboratory of Hebei Province, Shijiazhuang, China; ${ }^{4}$ Department of Neurology, Hebei General Hospital, \\ Shijiazhuang, China; ${ }^{5}$ Department of Neurosurgery, Hebei General Hospital, Shijiazhuang, China
}

"These authors contributed equally to this work.

Correspondence to: Kun Hong. Department of Neurology, the Second Hospital of Hebei Medical University, 215 Heping West Road, Shijiazhuang 050000, China. Email: hongkun999@163.com; Junying He. Department of Neurology, the Second Hospital of Hebei Medical University, 215 Heping West Road, Shijiazhuang 050000, China. Email: hjy_zn@126.com.

Submitted Jan 08, 2020. Accepted for publication Sep 24, 2020.

doi: 10.21037 /qims-20-45

View this article at: http://dx.doi.org/10.21037/qims-20-45

\section{Introduction}

Leptomeningeal carcinomatosis (LC) is still a devastating complication of cancer, and the incidence of $\mathrm{LC}$ in patients with solid tumors has increased in the past decade. The clinical presentation of LC depends primarily on the location of involvement and is variable and nonspecific. Median survival is low and is limited to 6-8 weeks without tumor-specific treatment. To date, the diagnosis of LC is still challenging. The diagnostic evaluation of suspected LC is on clinical findings, cerebrospinal fluid (CSF) analysis, and neuroimaging features (1). Although malignant cells in the CSF by cytology are considered the "gold standard", it is usually a qualitative analysis with low sensitivity as up to $30-50 \%$ of patients have a negative CSF result (2). Neuroimaging is an imperative procedure for detecting LC; about $70-80 \%$ of patients with leptomeningeal disease present abnormalities on image scans (3). Recently, symmetrical curvilinear cytotoxic edema along the surface of the brainstem has been considered a probable new magnetic resonance imaging (MRI) finding of LC (4-7).

\section{Case presentation}

A 65 -year-old hypertensive man was hospitalized with progressive worsening of unsteady gait and lower extremity weakness for two weeks. There was no bowel or bladder involvement. In May 2016, he was diagnosed with an inoperable lung adenosquamous carcinoma in the right upper lobe confirmed by lymph node (LN) biopsy, which had invaded the lung hilum, mediastinum, and cervical $\mathrm{LN}$, for which he received five cycles of low dose density paclitaxel plus cisplatin (TP) chemotherapy. During these three years, he was systemically followed once every two months, and no abnormalities were noted on physical examination and blood tests, including tumor markers. The patient also underwent chest CT twice a year, and there were no increases in the severity of the lesions.

Upon admission to our neurologic department, his examination revealed normal higher mental functions and intact cranial nerve function. He had decreased deeptendon reflexes in both lower extremities as muscle strength was $3 / 5$ and $4 / 5$ in his left and right leg, respectively, and no large fiber sensory changes in his legs/arms were found.

Laboratory examination revealed mild anemia (hemoglobin $11.2 \mathrm{~g} / \mathrm{dL}$ ), serum biochemistry, HIV and hepatitis serology, rapid plasma reagin, thyroidstimulating hormone, and glycated hemoglobin levels were unremarkable. However, tumor markers: serum carbohydrate antigen 153 , carbohydrate antigen 125 , carcinoembryonic antigen, and ferritin levels were mildly 

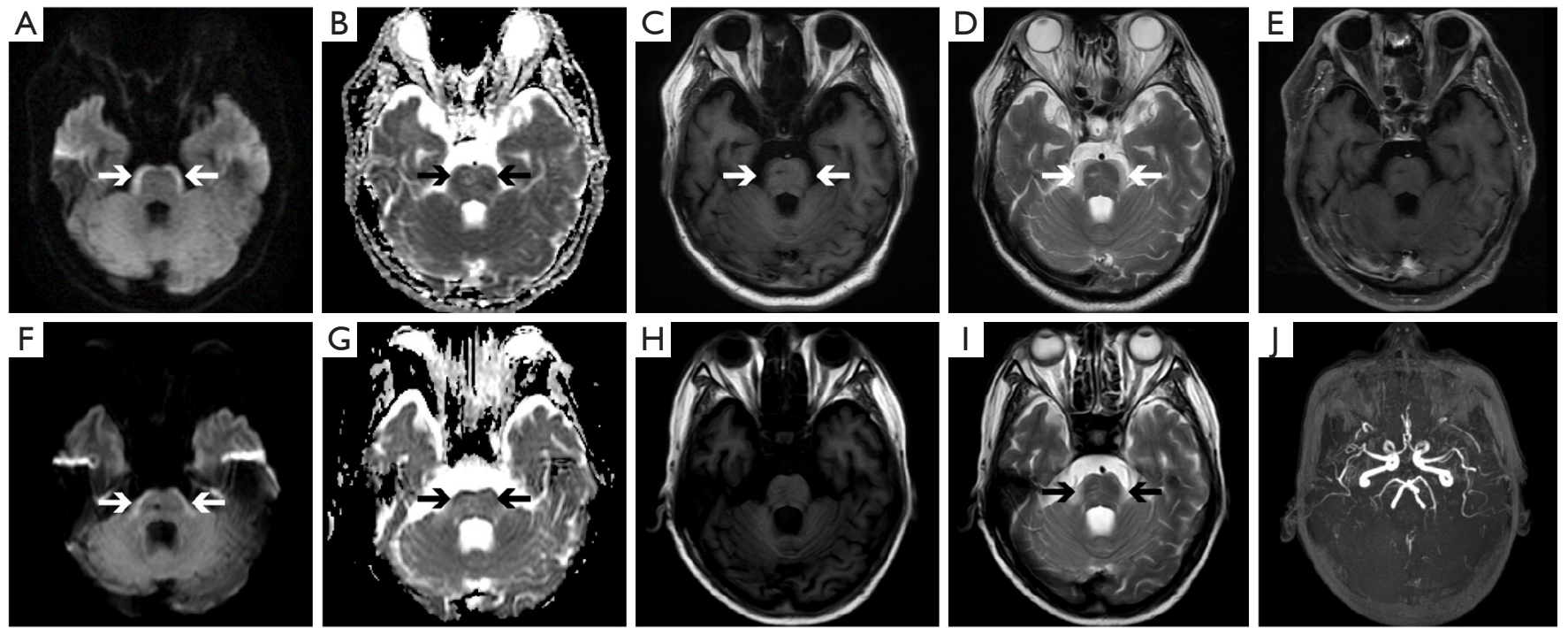

Figure 1 Brain magnetic resonance imaging (MRI) findings. Diffusion-weighted imaging (DWI)-, apparent diffusion coefficient (ADC), T1-, and T2-weighted images at first presentation and after intrathecal (IT) therapy is presented. Brain MR examination showed atypical findings of leptomeningeal carcinomatosis (LC) with the pons and middle cerebellar peduncle anterior surface DWI hyperintensities (A), ADC hypointensities (B), T1-weighted hypointensities (C), and T2-weighted hyperintensities (D). The lesions showed no enhancement (E). Brain MR re-examination showed the shrinkage of the LC-related DWI hyperintensities (F), low signal on ADC (G), the equal signal on T1-weighted imaging $(\mathrm{H})$, and high signal on T2-weighted imaging (I) along the anterior surface of the brainstem and the middle cerebellar peduncle. Time-of-flight magnetic resonance angiography (TOF-MRA) (J) only demonstrated mild atherosclerosis. The black or white arrows indicated the lesions.

elevated. Brain MRI reveals a symmetric curvilinear apparent low signal on T1-weighted imaging (T1WI) and apparent diffusion coefficient (ADC), high signal on T2weighted imaging (T2WI) and diffusion-weighted imaging (DWI), insufficient enhancement along the anterior surface of the pons, medulla, and the middle cerebellar peduncle (Figure 1A,B,C,D,E). Lumbar puncture with $\mathrm{CSF}$ analysis was performed with an opening pressure of $\geq 33 \mathrm{cmH}_{2} \mathrm{O}$, which revealed a normal cell count, a mildly elevated protein concentration $(0.44 \mathrm{~g} / \mathrm{L})$, and a decrease in glucose level $(1.73 \mathrm{mmol} / \mathrm{L})$. CSF cytology shows large, atypical cells suggestive of $\mathrm{LC}$. All investigated autoimmune encephalitis antibodies are negative in the serum and CSF. Given the poor prognosis of the patient, his family chose a conservative treatment, and he was discharged from the hospital.

However, after only six weeks, he was re-hospitalized owing to episodic headaches and dysarthria. Four doses in methotrexate and dexamethasone were administered by intrathecal therapy (IT), at a frequency of twice per week. Initially, the patient improved clinically with the regression of headaches, followed by improved speech and awareness.
Correspondingly, follow-up MRI showed shrinkage of brainstem lesions, characterized by hyperintensity on DWI and hypointense on ADC maps consistent with cytotoxic edema (Figure 1F,G,H,I, 手). Unfortunately, the patient died two weeks later because of an extensive tumor burden.

\section{Discussion}

LC is a devastating complication of nearly all systemic tumors. It has been reported in approximately $10 \%$ of patients with metastatic cancer in the disease's course $(1,3)$. Recent advances in systemic chemotherapy and targeted therapy have markedly improved the clinical outcomes in patients with LC $(8,9)$. Herein we describe a case of LC of adenosquamous lung carcinoma with features of the pons, medulla, and middle cerebellar peduncle anterior surface symmetrical curvilinear cytotoxic edema without enhancement, which was recognized as a new LC MRI pattern in 2014 for the first time (4). We retrospectively analyzed the details of patients with these characteristic brainstem MRI findings, as reported in the literature. Three of the nine cases developed parenchymal brain metastases 
associated or preceded by atypical brainstem MRI findings (4-7). The etiology and pathogenesis of this phenomenon remain unclear. Some researchers have hypothesized that malignant cells in the subarachnoid cisterns infiltrate the perforating arteries along the brainstem surface and cause microinfarctions $(5,6)$. Because of microvascular obstruction and insufficient collateral circulation, it is difficult for gadolinium to reach the brainstem lesions and so the lesions are usually not enhanced.

It is well-known that neuroimaging is a fundamental element in the diagnosis and response evaluation for patients with LC. Characteristic MRI abnormalities of LC include focal or diffuse sulcal enhancement, linear ependymal enhancement, cranial nerve or nerve root enhancement, leptomeningeal enhancing nodules of the brain or spinal cord, and hydrocephalus $(1,3,10)$. Because of the absence of these radiographic features typical of LC in our case, the scorecard for radiographic assessment is unsuitable for evaluating the treatment effects (1). In effect, after regular IT chemotherapy via lumbar puncture, the patient's symptoms of dysarthria decreased, and his mental state improved rapidly. Concurrently, the T2 hyperintense signal of the ventral surface of the pons persisted but was markedly reduced compared with the pretreatment evaluation. We hypothesize that T2 abnormalities without enhancement could also be considered a response determination in LC according to some circumstances, even if it has a false-positive possibility. Similarly, Cheng et al. (6) also reported that follow-up brain MRI showed persistent hyperintensity on fluid-attenuated inversion recovery, imaging, and restricted diffusion of the anterior brainstem lesions after IT chemotherapy. Although the proposal for response evaluation remains controversial because of its complexity, we support the view that a complete cerebrospinal MRI should be routinely repeated to evaluate response to treatment.

In conclusion, our case report highlights LC from adenosquamous lung carcinoma presents the uncommon but characteristic MRI finding of symmetric hyperintensity on DWI and hypointensity on ADC of a non-enhancing lesion along the surface of the ventral brainstem. To our knowledge, interestingly, only lung cancer as a primary tumor metastasizes to the leptomeninges and has similar MRI findings. At present, the pathway to understanding the pathological features associated with these imaging findings is not yet known. A larger number of cases will be needed to confirm these findings.

\section{Acknowledgments}

Funding: None.

\section{Footnote}

Conflicts of Interest: All authors have completed the ICMJE uniform disclosure form (available at http://dx.doi. org/10.21037/qims-20-45). Dr. HB serves as an unpaid editorial board member of Quantitative Imaging in Medicine and Surgery. The other authors have no conflicts of interest to declare.

Informed Consent: Written informed consent was obtained from the patient to publish this manuscript and accompanying images.

Open Access Statement: This is an Open Access article distributed in accordance with the Creative Commons Attribution-NonCommercial-NoDerivs 4.0 International License (CC BY-NC-ND 4.0), which permits the noncommercial replication and distribution of the article with the strict proviso that no changes or edits are made and the original work is properly cited (including links to both the formal publication through the relevant DOI and the license). See: https://creativecommons.org/licenses/by-nc-nd/4.0/.

\section{References}

1. Chamberlain M, Junck L, Brandsma D, Soffietti R, Rudà R, Raizer J, Boogerd W, Taillibert S, Groves MD, Le Rhun E, Walker J, van den Bent M, Wen PY, Jaeckle KA. Leptomeningeal metastases: a RANO proposal for response criteria. Neuro Oncol 2017;19:484-92.

2. Gauthier H, Guilhaume MN, Bidard FC, Pierga JY, Girre V, Cottu PH, Laurence V, Livartowski A, Mignot L, Diéras V. Survival of breast cancer patients with meningeal carcinomatosis. Ann Oncol 2010;21:2183-7.

3. Le Rhun E, Weller M, Brandsma D, Van den Bent M, de Azambuja E, Henriksson R, Boulanger T, Peters S, Watts C, Wick W, Wesseling P, Rudà R, Preusser M. CollectiveName: EANO Executive Board and ESMO Guidelines Committee. EANO-ESMO Clinical Practice Guidelines for diagnosis, treatment and follow-up of patients with leptomeningeal metastasis from solid tumours. Ann Oncol 2017;28:iv84-iv99.

4. Crombe A, Alberti N, Durieux M, Frulio N, Dousset V, Tourdias T. Exceptional symmetric anterior brainstem 
involvement in leptomeningeal carcinomatosis. J

Neuroradiol 2014;41:279-81.

5. Khil EK, Lee AL, Chang KH, Yun TJ, Hong HS.

Symmetrical Curvilinear Cytotoxic Edema Along the Surface of the Brain Stem: A Probable New Magnetic Resonance Imaging Finding of Leptomeningeal Carcinomatosis. Medicine (Baltimore) 2015;94:e1053.

6. Cheng CY, Hsu CY, Tsai YH, Lin KL, Huang CE, Fan YH, Chin SC, Huang YC. Novel Anterior Brainstem Magnetic Resonance Imaging Findings in Non-Small Cell Lung Cancer with Leptomeningeal Carcinomatosis. Front Neurol 2017;8:579.

7. Maeda K, Wada H, Ozaki Y, Inoue A. Symmetric Ventral Brainstem Lesion in Leptomeningeal Carcinomatosis. Intern Med 2019;58:759-60.

8. Scott BJ, van Vugt VA, Rush T, Brown T, Chen CC,

Cite this article as: Liu C, Zhang Y, Li Y, Bu H, Meng Z, Ji Y, Wu J, Zou Y, Chen X, He J, Hong K. A rare magnetic resonance imaging pattern of leptomeningeal carcinomatosis: a case description. Quant Imaging Med Surg 2021;11(7):3367-3370. doi: 10.21037/qims-20-45
Carter BS, Schwab R, Fanta P, Helsten T, Bazhenova L, Parker B, Pingle S, Saria MG, Brown BD, Piccioni DE, Kesari S. Concurrent intrathecal methotrexate and liposomal cytarabine for leptomeningeal metastasis from solid tumors: a retrospective cohort study. J Neurooncol 2014;119:361-8.

9. Dudani S, Mazzarello S, Hilton J, Hutton B, Vandermeer L, Fernandes R, Ibrahim MF, Smith S, Majeed H, AlBaimani K, Caudrelier JM, Shorr R, Clemons M. Optimal Management of Leptomeningeal Carcinomatosis in Breast Cancer Patients-A Systematic Review. Clin Breast Cancer 2016;16:456-70.

10. Chamberlain MC, Glantz M, Groves MD, Wilson WH. Diagnostic tools for neoplastic meningitis: detecting disease, identifying patient risk, and determining benefit of treatment. Semin Oncol 2009;36:S35-S45. 